\title{
Sistemas de Aterramiento con la Utilización de Conductores y Barras Envueltos en Concreto
}

\author{
Arnaldo G. Kanashiro y Sergio R. C. Bezerra \\ Universidade de São Paulo, Instituto de Eletrotécnica e Energia, Av. Prof. Luciano Gualberto, 1289. \\ CEP 05508-010, São Paulo - SP, Brasil. (e-mail: arnaldo@iee.usp.br) \\ Recibido Ago. 11, 2011; Aceptado Oct. 06, 2011; Versión final recibida Dic. 01, 2011
}

\begin{abstract}
Resumen
Este trabajo presenta los resultados de simulaciones computacionales considerándose la utilización de conductores y barras envueltos en concreto en sistemas de aterramiento. Se ha utilizado el programa computacional Current Distribution, Electromagnetic Fields, Grounding and Soil Structure Analysis (CDEGS) para la determinación de las resistencias de tierra. Se utilizaron valores típicos de resistividad del suelo y valores medidos de la resistencia de tierra, que fueron comparados posteriormente con resultados obtenidos en simulaciones computacionales. Los valores de la resistencia de tierra, considerándose el uso del concreto, se presentaron menores que aquellos obtenidos con sistemas de aterramiento utilizando conductores y barras convencionales. Los resultados de las simulaciones fueron próximos de aquellos encontrados en mediciones realizadas en campo.
\end{abstract}

Palabras clave: sistemas de aterramiento, resistencia de tierra, simulación computacional, CDEGS

\section{Grounding Systems using Concrete Encased Conductors and Electrodes}

\begin{abstract}
This work presents the results of computational simulations considering the use of concrete encased conductors and electrodes in grounding systems. The software tool Current Distribution, Electromagnetic Fields, Grounding and Soil Structure Analysis (CDEGS) was used for determining ground grid resistances. Typical values of soil resistivity and measured ground resistances were taken into account in this work to then compare them with the results obtained in the computational simulations. The results showed that the ground resistances considering concrete encased conductors and electrodes were lower than the values obtained with conventional grounding systems. The comparison between results of the computational simulations and measured values showed reasonable agreement.
\end{abstract}

Keywords: grounding systems, ground resistance, computational simulations, CDEGS 


\section{INTRODUCCIÓN}

La técnica de envolver barras de aterramiento en concreto fue creada por el ingeniero Helbert Ufer, durante la segunda guerra mundial (Fagan y Lee, 1970; Telló et al., 2007). Los Estados Unidos, en la época, necesitaban de un aterramiento confiable, en sus depósitos, para evitar la detonación de explosivos durante la ocurrencia de descargas atmosféricas (Piantini y Janiszewski, 2004; Grcev, 2009; Villa et al., 2009). Este tipo de aterramiento es conocido en la literatura como aterramiento Ufer. Los resultados de Ufer consideran que el metal encapsulado con concreto actúa como un electrodo de aterramiento eficaz (Khalid et al., 2011). El concreto es un material utilizado en la construcción civil, compuesto de una mezcla de cemento, arena, piedras y agua, además de otros materiales eventuales, tales como aditivos. El concreto es higroscópico, o sea, absorbe humedad rápidamente y la pierde lentamente. Un bloque de concreto enterrado en el suelo se comporta como un semiconductor, con resistividad variando entre 30 e $90 \Omega$.m (IEEE Std $80,2000)$.

Para una barra convencional, el valor de la resistencia de tierra puede ser obtenido por la expresión (1):

$$
R_{a t}=\frac{\rho_{a}}{2 \pi \times L} \ln \left(\frac{4 L}{d}\right)
$$

donde: $R_{a t}=$ resistencia de tierra $(\Omega) ; \rho_{a}=$ resistividad aparente del suelo $(\Omega . m)$; L=longitud de la barra $(\mathrm{m}) ; \mathrm{D}=$ diámetro de la barra $(\mathrm{m})$

La expresión (2) puede ser utilizada para calcular el valor de la resistencia de tierra de una barra de aterramiento envuelta en concreto (IEEE Std 80, 2000):

$$
R_{C E}=\frac{1}{2 \pi \times L_{r}}\left\{\rho_{c}\left[\ln \left(D_{c} / d\right)\right]+\rho\left[\ln \left(8 L_{r} / D_{c}\right)-1\right]\right\}
$$

donde: $R_{C E}=$ resistencia de tierra $(\Omega) ; \rho=$ resistividad del suelo $(\Omega . m) ; \rho_{c}=$ resistividad del concreto $(\Omega . m) ; d=$ diámetro de la barra $(m) ; D_{c}=$ diámetro del encapsulamiento de concreto $(m) ; L_{r}=$ longitud de la barra $(m)$

En Clausen et al. (2004) fue realizada una investigación experimental considerándose mallas convencionales y con barras y conductores envueltos en concreto. Fueron utilizados barras de cobre como base para la construcción de la mayoría de las mallas. En la primera etapa de la investigación, fueron consideradas cinco configuraciones: 1 barra simple; 3 y 5 barras en línea; 3 barras en $\mathrm{V}$ y 3 barras en triángulo. El local donde estas mallas fueron construidas posee suelo homogéneo, del tipo arenoso oscuro, con resistividad alrededor de $1000 \Omega$.m. A efecto de comparación, fueron construidas dos configuraciones con las mismas características citadas anteriormente, siendo con 1 barra simple y 3 barras en línea, todos envueltos en concreto, en la proporción de cinco porciones de arena gruesa para una de cemento. Después del uso del concreto, las barras y los conductores quedaron con diámetro de $40 \mathrm{~cm}$. La reducción de resistencia de tierra después, con el concreto, fue de $38 \%$ en relación a la convencional. En el caso de la malla con tres barras alineados, la reducción fue de $33,9 \%$ con o uso del concreto. La Fig. 1 muestra una malla con conductores y barras envueltos en concreto.

De este modo, los autores (Clausen et al., 2004) presentaron resultados de mediciones que posibilitaron la proposición de una nueva técnica de aterramiento para sistemas de distribución de energía, o sea, la utilización del propio poste duplo "T" como parte del aterramiento eléctrico, pues con el uso de las barras concretadas e de los propios postes, los resultados medidos en campo fueron excelentes. En Souza et al. (2007) fueron analizadas alternativas para la construcción de mallas de aterramiento en un suelo con alta resistividad eléctrica, con base en resultados obtenidos en mediciones. 


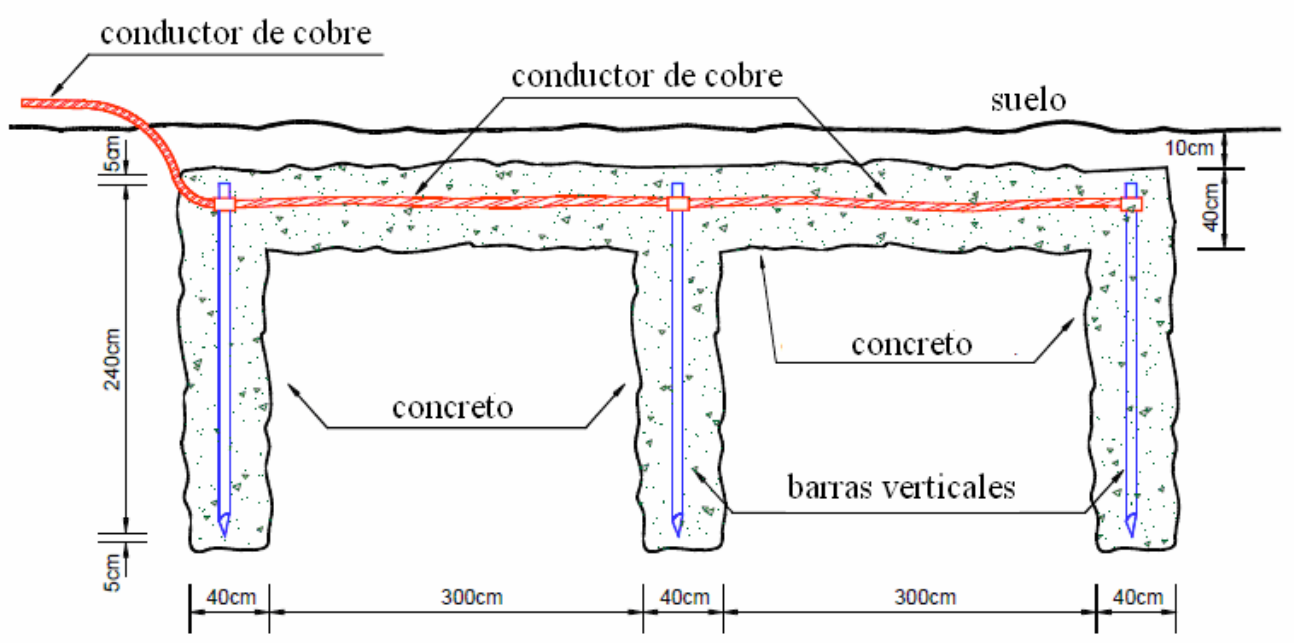

Fig. 1: Malla con conductores y barras envueltos en concreto (Clausen et al., 2004)

En la primera etapa del trabajo, fueron construidas dos mallas en triángulo, una con barras convencionales y otra con barras concretadas. La malla utilizando barras concretadas fue construida con la misma mezcla de concreto que es usada para construcción de postes de las redes de distribución de energía eléctrica. Después de la construcción de las dos mallas, fueron iniciadas las mediciones a través de la utilización de un terrómetro digital de cuatro puntas (Devarakonda et al., 2007). Los valores de resistencia de tierra fueron obtenidos durante dos períodos (lluvioso y seco). El aterramiento que utilizó barras concretadas presentó valores de resistencia de tierra significativamente menores cuando comparados con la malla de aterramiento convencional. Para el período seco, la malla con barras concretadas presentó reducción de $61 \%$ del valor de resistencia de tierra en comparación con el valor de la malla convencional. El valor de resistencia para el período lluvioso presentó reducción de $34 \%$ en relación a la malla convencional.

Los resultados mostrados en Clausen et al. (2004) y Souza et al. (2007) fueron obtenidos a través de mediciones en el campo, sin que se realizaran comparaciones con cálculos teóricos de las resistencias de tierra. Este trabajo presenta los resultados de simulaciones computacionales de configuraciones de aterramiento considerándose conductores y barras convencionales y envueltos en concreto. Inicialmente, fueron estudiadas configuraciones simples, con el objetivo de se analizar la influencia del barra de concreto en relación al barra convencional. En seguida, fueron consideradas las configuraciones utilizadas en Clausen et al. (2004) con el objetivo de comparar las mediciones realizadas en campo con los resultados obtenidos en simulaciones computacionales.

\section{METODOLOGÍA}

El programa computacional Current Distribution, Electromagnetic Fields, Grounding and Soil Structure Analysis (CDEGS, 2000) es un programa de origen canadiense que permite el análisis de la resistividad del suelo, a través de la estratificación del suelo por camadas, y de sistemas de aterramiento de subestaciones, redes de distribución y líneas de transmisión. El dominio de este recurso ayuda a dimensionar y optimizar adecuadamente los sistemas de aterramiento, con el objetivo de resolver una variedad de problemas. Esta herramienta posee paquetes de ingeniería, tales como: RESAP, MALT y el MALZ. Los paquetes presentados poseen características específicas, siendo el último abocado hacia el desarrollo de este trabajo. Por tanto, van a ser presentadas las características de este paquete. Los datos básicos a ser propiciados para la simulación a través del paquete MALZ son: suelo (tipo, resistividad y disposición de camadas) y configuración de las barras (posición física de los conductores). El programa MALZ es una herramienta de análisis de sistemas de aterramiento en el dominio de la frecuencia, adecuado 
para determinar corrientes de fuga, campos eléctricos y magnéticos generados por una red de conductores enterrados en el suelo, y potenciales en el suelo, tomándose en consideración todas las inductancias y capacitancias internas y su localización en la malla de aterramiento. EI MALZ puede ser utilizado para analizar conductores revestidos con resistividad y espesor definidos por el usuario. Esta fue una de las principales ventajas del programa, donde se pudo envolver la barra en concreto, utilizando un valor típico de resistividad del concreto. Las Fig. 2 y 3 muestran las mallas de aterramiento iniciales consideradas en este trabajo.

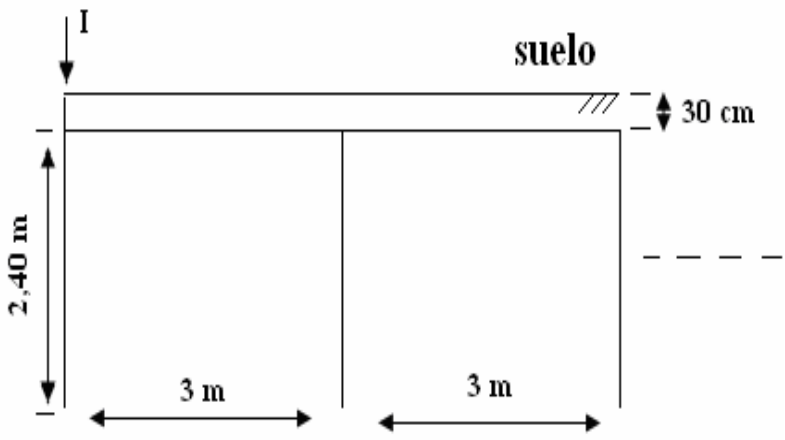

Fig. 2: Malla convencional con barras y conductores alineados

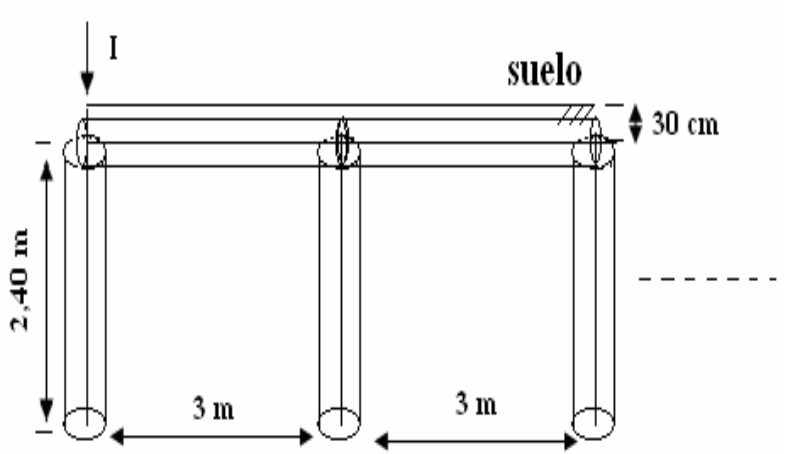

Fig. 3: Malla con barras y conductores concretados

Los siguientes pasos fueron adoptados para el desarrollo de este trabajo: a) Configuración de la malla de aterramiento convencional con el uso de barras de cobre; b) Configuración de la malla de aterramiento con barras y conductores de cobre envueltos en concreto, con las mismas características de la malla convencional; c) Simulación computacional de las mallas de aterramiento, (a) e (b), para obtención del valor de resistencia de tierra. El suelo fue considerado homogéneo para todas las simulaciones; d) Simulación computacional de la malla de aterramiento convencional y malla con barras y conductores envueltos en concreto.

\section{RESULTADOS Y ANALISIS}

Los resultados presentados en las Tablas 1 a 4 son referentes a las configuraciones de las mallas de aterramiento convencionales y concretadas, mostradas en las Fig. 2 y 3 . Las barras de cobre son colocadas en la posición vertical, $30 \mathrm{~cm}$ abajo de la superficie del suelo y su interconexión se hace con cabos en la posición horizontal. Las barras son cilíndricas y cobreadas, con diámetro de $12,5 \mathrm{~mm}$ y $2,4 \mathrm{~m}$ de longitud. El espaciamiento inicial entre barras es de $3 \mathrm{~m}$. Las interconexiones de las barras se hacen a través de conductores de cobre desnudos, con $25 \mathrm{~mm}^{2}$ de diámetro. Los cálculos se efectuaron tomándose en consideración el valor de resistividad media $60 \Omega$.m para el concreto (IEEE Std 80,2000 ) y diámetro de $20 \mathrm{~cm}$. Las simulaciones fueron realizadas considerándose la frecuencia industrial de $60 \mathrm{~Hz}$. En la Tabla 6 son mostradas simulaciones considerándose hasta 12 barras, tanto para la malla convencional como para la malla envuelta en concreto. De esta forma, se observa que también existe una saturación en la disminución de la resistencia de tierra con el aumento del número de barras. A continuación, son presentados, en las Fig. 4 a 6, los valores de las resistencias de tierra referentes a una barra y barras alineadas ( 2 a 6 barras), con resistividades del suelo de 100, 1000 y $3000 \Omega$.m. La Fig. 4 presenta los resultados de 1 a 6 barras en línea $\left(\rho_{\text {suelo }} 100 \Omega\right.$.m). Con una barra, se observa reducción de $15,5 \%$ en el valor de la resistencia de tierra, cuando es utilizado el concreto. Con el clavar del segundo barra, fue obtenida reducción de $11,6 \%$. Cuando se aumenta el número de barras, los valores de resistencia de tierra entre la barra convencional y la barra concretada son próximos. Se resalta que la resistividad del concreto es del mismo orden de grandeza que la resistividad del suelo.

Tabla 1: Valores de resistencia de tierra (1 y 2 barras) 


\begin{tabular}{|c|c|c|c|c|}
\hline Configuración de la malla & $\mathrm{N}^{\circ}$ de barras & $\rho_{\text {suelo }}(\Omega \cdot \mathrm{m})$ & Rat convencional $(\Omega)$ & Rat concreto $(\Omega)$ \\
\hline barra simple & 1,0 & 100 & 36,7 & 31,2 \\
\hline barras alineadas & 2,0 & 100 & 19,0 & 16,8 \\
\hline barras alineadas & 2,0 & 500 & 92,6 & 67,4 \\
\hline barras alineadas & 2,0 & 1.000 & 184,6 & 130,2 \\
\hline barras alineadas & 2,0 & 2.000 & 368,7 & 255,8 \\
\hline barras alineadas & 2,0 & 3.000 & 552,7 & 381,3 \\
\hline
\end{tabular}

Tabla 2: Valores de resistencia de tierra (3 barras)

\begin{tabular}{|c|c|c|c|c|}
\hline Configuración de la malla & $\mathrm{N}^{\circ}$ de barras & $\rho_{\text {suelo }}(\Omega . \mathrm{m})$ & Rat convencional $(\Omega)$ & Rat concreto $(\Omega)$ \\
\hline barras alineadas & 3 & 100 & 13,9 & 12,6 \\
\hline barras alineadas & 3 & 500 & 65,6 & 50,0 \\
\hline barras alineadas & 3 & 1.000 & 130,1 & 96,4 \\
\hline barras alineadas & 3 & 2.000 & 259,2 & 189,4 \\
\hline barras alineadas & 3 & 3.000 & 388,3 & 281,8 \\
\hline
\end{tabular}

Tabla 3: Valores de resistencia de tierra (4 barras)

\begin{tabular}{|c|c|c|c|c|}
\hline Configuración de la malla & $\mathrm{N}^{\circ}$ de barras & $\rho_{\text {suelo }}(\Omega . \mathrm{m})$ & Rat convencional $(\Omega)$ & Rat concreto $(\Omega)$ \\
\hline barras alineadas & 4,0 & 100 & 11,6 & 10,7 \\
\hline barras alineadas & 4,0 & 500 & 52,2 & 40,9 \\
\hline barras alineadas & 4,0 & 1.000 & 102,9 & 78,4 \\
\hline barras alineadas & 4,0 & 2.000 & 204,2 & 153,4 \\
\hline barras alineadas & 4,0 & 3.000 & 305,6 & 228,4 \\
\hline
\end{tabular}

Tabla 4: Valores de resistencia de tierra (5 barras)

\begin{tabular}{|c|c|c|c|c|}
\hline Configuración de la malla & $\mathrm{N}^{\circ}$ de barras & $\left.\rho_{\text {suelo }} \Omega \cdot \mathrm{m}\right)$ & Rat convencional $(\Omega)$ & Rat concreto $(\Omega)$ \\
\hline barras alineadas & 5,00 & 100 & 10,3 & 9,6 \\
\hline barras alineadas & 5,00 & 500 & 44,2 & 35,3 \\
\hline barras alineadas & 5,00 & 1.000 & 86,3 & 67,2 \\
\hline barras alineadas & 5,00 & 2.000 & 170,6 & 130,8 \\
\hline barras alineadas & 5,00 & 3.000 & 254,9 & 194,3 \\
\hline
\end{tabular}

Tabla 5: Valores de resistencia de tierra (6 barras)

\begin{tabular}{|c|c|c|c|c|}
\hline Configuración de la malla & $\mathrm{N}^{\circ}$ de barras & $\rho_{\text {suelo }}(\Omega . \mathrm{m})$ & Rat convencional $(\Omega)$ & Rat concreto $(\Omega)$ \\
\hline barras alineadas & 6,00 & 100 & 9,61 & 8,9 \\
\hline barras alineadas & 6,00 & 500 & 38,8 & 31,5 \\
\hline barras alineadas & 6,00 & 1.000 & 75,2 & 59,4 \\
\hline barras alineadas & 6,00 & 2.000 & 147,7 & 114,9 \\
\hline barras alineadas & 6,00 & 3.000 & 220,3 & 170,5 \\
\hline
\end{tabular}

Tabla 6: Valores de resistencia de tierra (7 a 12 barras)

\begin{tabular}{|c|c|c|c|c|}
\hline Configuración de la malla & $\mathrm{N}^{\circ}$ de barras & $\rho_{\text {suelo }}(\Omega . \mathrm{m})$ & Rat convencional $(\Omega)$ & Rat concreto $(\Omega)$ \\
\hline barras alineadas & 7,0 & 3000 & 193,4 & 152,2 \\
\hline barras alineadas & 8,0 & 3000 & 175,8 & 139,0 \\
\hline barras alineadas & 9,0 & 3000 & 160,0 & 128,0 \\
\hline
\end{tabular}




\begin{tabular}{|c|c|c|c|c|}
\hline barras alineadas & 10,0 & 3000 & 148,2 & 119,0 \\
\hline barras alineadas & 11,0 & 3000 & 138,0 & 111,0 \\
\hline barras alineadas & 12,0 & 3000 & 129,4 & 105,0 \\
\hline
\end{tabular}

La Fig. 5 muestra que el valor de la resistencia de tierra fue reducido en $29,5 \%$ con la utilización de dos barras concretadas. Comparándose el valor con tres barras concretadas, hubo reducción de $25,9 \%$ y, cuando se aumenta el número de barras, la diferencia entre los valores disminuye. Se observa en la Fig. 6, que el valor de resistencia de tierra se redujo en 31,0\% con la utilización de dos barras concretadas. No obstante, con la tercera barra hubo reducción de $27,4 \%$. Cuando se compara el valor de resistencia de tierra de la malla, alterándose solamente los valores de resistividad del suelo, se observa que con mayor resistividad del suelo, hay una reducción más significativa en la resistencia de tierra de la malla con la utilización del barra envuelta en concreto, volviéndose más eficiente el uso del mismo en suelos con resistividades elevadas.

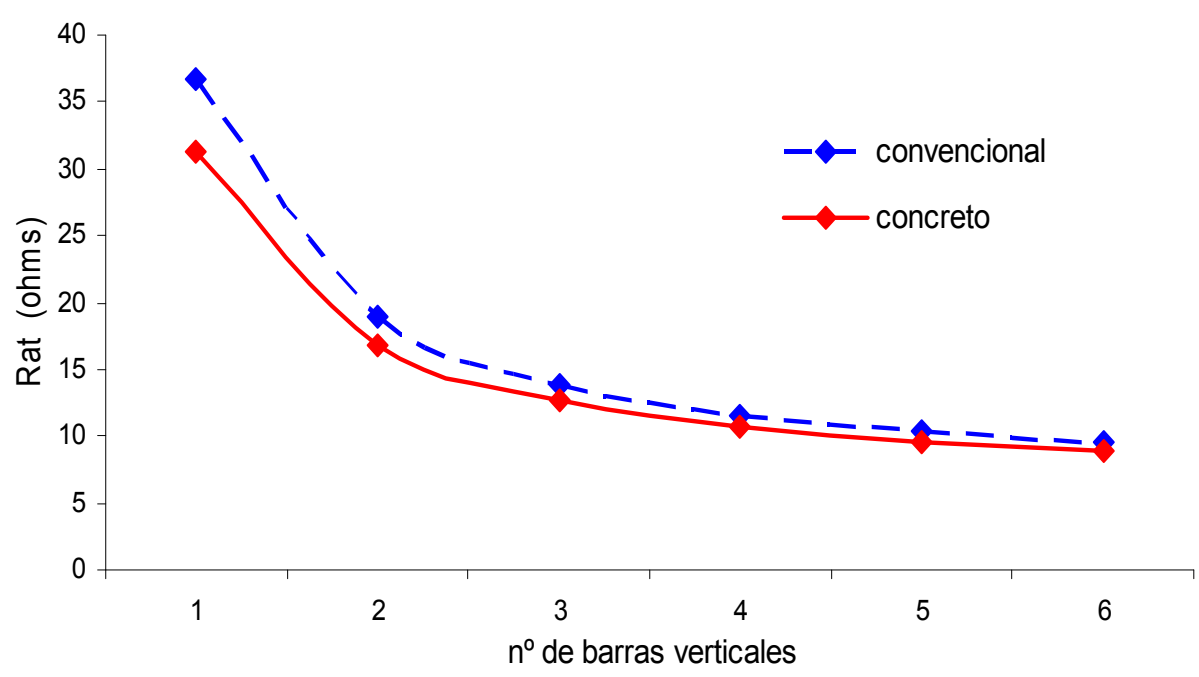

Fig. 4: Malla convencional vs. malla concretada ( $\rho_{\text {suelo }} 100 \Omega . m$ )

La Tabla 7 muestra los valores de la resistencia de tierra medidos en campo (Clausen et al., 2004) y los resultados de simulaciones realizadas con el programa computacional CDEGS. Las configuraciones de las mallas son constituidas de barras cilíndricas cobreadas, con diámetro de $13 \mathrm{~mm}$ y $2,4 \mathrm{~m}$ de longitud, clavadas en la vertical, a $30 \mathrm{~cm}$ por debajo de la superficie del suelo y espaciamiento entre barras de $3 \mathrm{~m}$. Las interconexiones de las barras, cuando necesario, se hacen a través de cabos desnudos, con $25 \mathrm{~mm}^{2}$ de sección, en la posición horizontal. Las simulaciones fueron efectuadas, considerándose suelo homogéneo, con resistividad de $1000 \Omega$.m, o sea, el mismo valor mencionado en Clausen et al. (2004). 


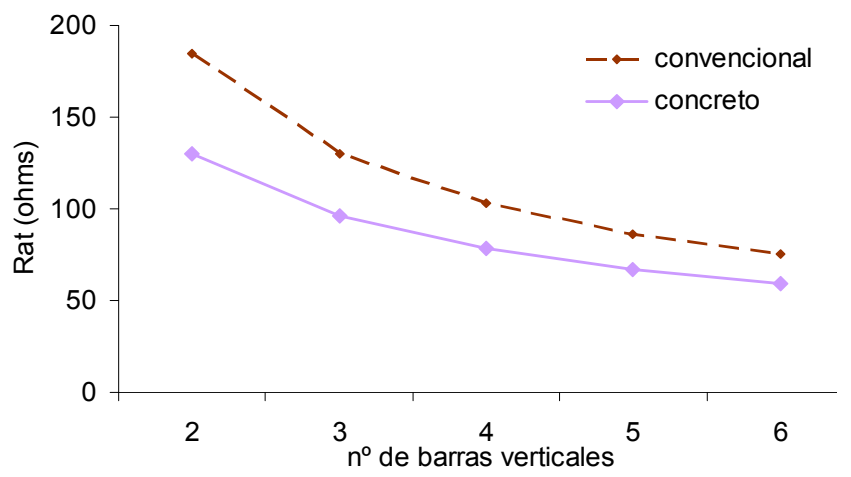

Fig. 5: Malla convencional vs. malla concretada $\left(\rho_{\text {suelo }} 1000 \Omega . m\right)$

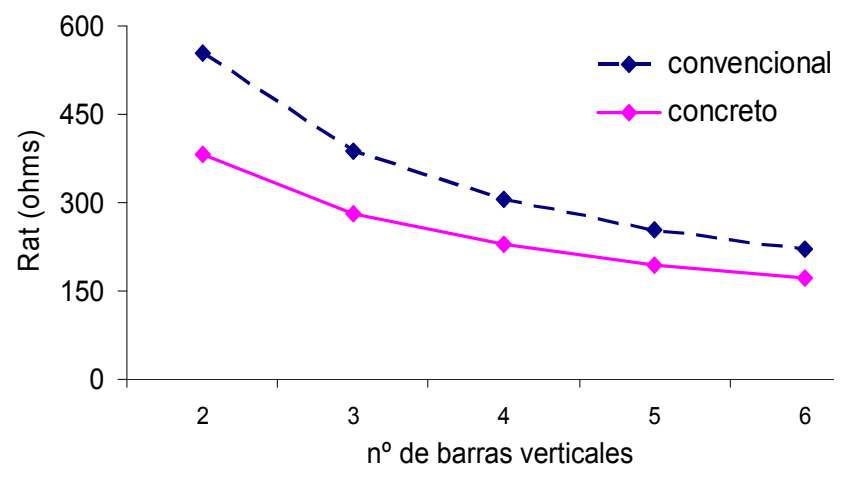

Fig. 6: Malla convencional vs.malla concretada ( $\rho_{\text {suelo }} 3000 \Omega . m$ )

Tabla 7: Valores de la resistencia de tierra medidos en campo y obtenidos en las simulaciones

\begin{tabular}{|c|c|c|c|c|}
\hline $\begin{array}{c}\text { Configuración de } \\
\text { la malla }\end{array}$ & $\begin{array}{c}\text { Rat convencional } \\
\text { CDEGS }-(\Omega)\end{array}$ & $\begin{array}{c}\text { Rat convencional - } \\
\text { medido }-(\Omega)\end{array}$ & $\begin{array}{c}\text { Rat concreto- } \\
\text { CDEGS }-(\Omega)\end{array}$ & $\begin{array}{c}\text { Rat concreto- } \\
\text { medido }-(\Omega)\end{array}$ \\
\hline barra simple & 402,00 & 392,3 & 203,90 & 201,70 \\
\hline 3 barras alineadas & 130,00 & 134,70 & 84,00 & 80,00 \\
\hline 5 barras alineadas & 84,00 & 118,30 & 58,75 & Sin medición \\
\hline En "V" & 136,44 & 169,70 & 92,53 & Sin medición \\
\hline Triángulo & 130,00 & 160,00 & 89,50 & Sin medición \\
\hline
\end{tabular}

Para el concreto, fue adoptado el valor de resistividad media igual a $60 \Omega$.m y utilizado el diámetro de $40 \mathrm{~cm}$ (Clausen y et al., 2004). Todas las configuraciones de aterramiento fueron simuladas considerándose la frecuencia industrial de $60 \mathrm{~Hz}$. A pesar de no disponerse de un mayor número de mediciones en campo, la utilización de barras envueltas en concreto resultó en menores valores de la resistencia de tierra, siendo la reducción más pronunciada para suelos de resistividades más elevadas. Para una barra simple envuelta en concreto fue observada una diferencia de $1,1 \%$. Cuando son considerados 3 barras envueltas en concreto, alineadas y espaciadas entre sí por 3 metros, fue obtenida una diferencia de $4,8 \%$ entre el valor medido en campo y el valor calculado. Los valores de la resistencia de tierra para las configuraciones con barras convencionales presentaron desvíos porcentuales de $2,4 \%$ hasta $29,0 \%$.

\section{CONCLUSIONES}

De los resultados de simulaciones computacionales de mallas de aterramiento considerándose barras y conductores convencionales y envueltos en concreto, se obtienen las siguientes conclusiones:

i) utilización de barras y conductores envueltos en concreto resultó en menores valores de la resistencia de tierra, siendo la reducción más pronunciada para suelos de resistividades más elevadas;

ii) a barras y conductores envueltos en concreto, los valores de la resistencia de tierra obtenidos en las simulaciones computacionales y las mediciones realizadas en campo presentaron desvíos de $1,1 \%$ (1 barra) y $4,8 \%$ (3 barras); 
iii) relación a los conductores y barras convencionales, fueron observados desvíos de $2,4 \%$ a $29,0 \%$.

\section{AGRADECIMIENTOS}

Los autores agradecen a la Coordenação de Aperfeiçoamento de Pessoal de Nível Superior (CAPES), por el apoyo financiero a través de concesión de beca de postgrado.

\section{REFERENCIAS}

CDEGS, User's Primers: Current Distribution, Electromagnetic Interference, Grounding and Soil Structure Analysis - SES Safe Engineering Services \& Technologies Ltd, Laval, Canada (2000).

Clausen, W. y otros seis autores, "Nova Técnica de Aterramento para Sistemas de Distribuição de Energia", XVI Seminário Nacional de Distribuição de Energia Elétrica (XVI SENDI), Brasília, Brasil, Nov. 21-24 (2004).

Devarakonda, L. S. y otros dos autores, "Evaluation of Ground Grid Resistance for Inservice Substations", Transmission and Distribution Conference and Exposition (2010 IEEE PES), 1-4, New Orleans, April 19-22 (2010).

Fagan, E. J. y R. H. Lee, The Use of Concrete-Enclosed Reinforcing Rods as Grounding Electrodes, IEEE Transactions on Power Delivery, 6 (4), 337-348 (1970).

Grcev L., Impulse Efficiency of Ground Electrodes, IEEE Transactions on Power Delivery, 24 (1), 441-450 (2009).

IEEE Std 80, IEEE Guide for Safety in AC Substation Grounding, 192, New York, USA (2000).

Khalid, A. y otros dos autores, "Study on the Usage of Structural Building as Main Lightning Protection", The $5^{\text {th }}$ International Power Engineering and Optimization Conference (PEOCO2011), 432-435, Selangor, June 6-7 (2011).

Piantini, A. y J. M. Janiszewski, Efectos de las Ramificaciones y de los Edificios en los Voltajes Inducidos por Rayos en Líneas de Distribución Urbanas, Información Tecnológica, 15 (6), 51-58 (2004).

Souza, K. y otros dos autores, "Estudo de Alternativas para a Construção de Malhas de Aterramento num Solo com Alta Resistividade Elétrica", II Congresso de Pesquisa e Inovação da Rede Norte Nordeste de Educação Tecnológica (II CONNEPI), João Pessoa, Brasil, Nov. 27-30 (2007).

Telló, M. y otros cinco autores, "Aterramento Elétrico Impulsivo, em Baixa e Alta Frequencias", $1^{\text {a }}$ ed., 328, EDIPUCRS, Porto Alegre, Brasil (2007).

Villa, W. M. y otros tres autores, Calculo del Campo Magnético en el Interior de Edificaciones con Sistemas de Protección Contra Rayos, Información Tecnológica, 20 (3), 45-54 (2009). 